\title{
Classroom experiments on laser physics
}

\author{
Alika Khare
}

Alika Khare, "Classroom experiments on laser physics," Proc. SPIE 9664, Ninth International Topical Meeting on Education and Training in Optics and Photonics, 966412 (24 October 2005); doi: 10.1117/12.2207534 
Ref ETOP : ETOP004

Class Room Experiments on Laser Physics

Alika Khare

Department of Physics

Indian Institute of Technology, Guwahati, Guwahati, 781039, India

email: alika@iitg.ernet.in

\begin{abstract}
Lasers have made the revolution in the modern optical technologies but there is hardly any efforts to take up the basic understanding of laser Physics via laboratory classes at graduate and undergraduate level. In this paper, a simple experiment for studying the life time of the upper laser level under lasing condition, the relaxation oscillations, measurement of threshold current and variation of laser power as a function of current in a laser diode is presented. The experiment utilizes the readily available low cost components, a key chain laser and some of the electronics instrument normally available at any undergraduate laboratory of science and engineering department.
\end{abstract}

\title{
Key words
}

class room experiment on laser physics, relaxation oscillations

\section{Introduction}

\section{Summary}

Optics is playing a leading role towards improving the day to day technologies in terms of precision and speed. This is possible with the extensive use of laser. Lasers have also become an essential tool now a days in any teaching laboratory at graduate and under graduate level. However experiments demonstrating some of the basic laser physics phenomena's are normally missing from the teaching laboratory. In this paper, simple experiments for studying the life time of the upper laser level under lasing condition, the relaxation oscillations, measurement of threshold current, variation of laser power as a function of current and the photon life time in a laser diode is presented. These concepts not only improve the understanding of the laser physics but also helps the student in realizing the range in which a given laser system can be used efficiently. The experiments utilize the readily available low cost components, a key chain laser and some of the electronics instrument normally available at any undergraduate laboratory of science and engineering department. The key chain laser is to be removed from its outer capsule carefully so as to connect it to the various circuits discussed below.

\section{Experimental set-up}

The first step in operating a laser is to determine the threshold pump and its variation with the pump. In case of diode laser, the pump is in the form of forward biased current. For this, the diode laser was operated in cw mode by forward biasing it with a dc power supply as shown in fig 1a. The variation in laser power was recorded as a function of current by a power meter or a photodiode (operated in photo conducting mode) as shown in fig1 $\mathrm{b}$. The current at which the laser output suddenly increases corresponds to the threshold current for the onset of laser oscillations. While modulating the laser with 
an external source, for pulsed mode, the dc current through the laser has to be maintained sufficiently above this threshold value so that laser is never driven below the threshold ${ }^{1}$. The phenomena of damped oscillations in the laser output around steady state is termed as relaxation oscillations ${ }^{1,2}$. It restricts the modulation frequency of a diode laser and thus limits the bandwidth of systems based on such lasers.

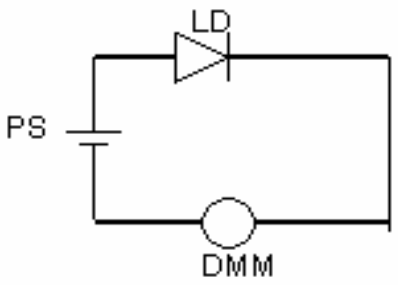

a

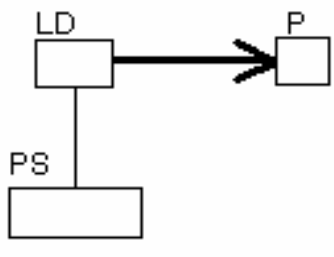

b

Fig 1. Experimental set-up for recording the variation of laser power as function of current, a. circuit diagram, b. block diagram. LD; Laser diode, PS; dc power supply, DMM; digital millimeter and $\mathrm{P}$; power meter,

To measure the relaxation oscillations, the diode laser operating under steady state was modulated by injecting the additional current pulse in the form of square wave from a simple circuit ${ }^{1}$. A square wave signal from the function generator is applied to the current amplifier $^{3}$ and the output of the current amplifier is then fed on to the diode laser (already maintained above threshold) through a Schmitt trigger ${ }^{1}$. Whenever output of the trigger is low, current through the diode laser will be more and corresponds to step pump. The sudden jump in the current (pump) results into relaxation oscillations in the laser output. If the pulse duration is sufficiently large then the laser will be driven to another steady state condition during this pump pulse.

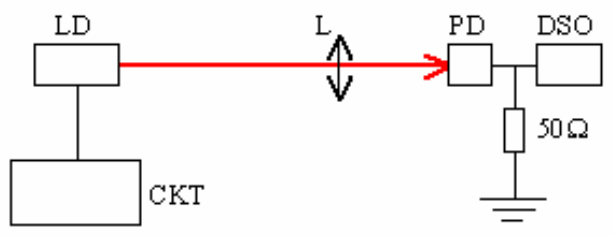

Fig 2. Schematic of the experimental set-up for recording relaxation oscillations, LD: laser diode, CKT circuit for the pump modulation, L; focusing lens, PD photodiode, DSO; digital storage oscilloscope.

The modulated laser beam was focused on to the photodiode through a convex lens. The photodiode signal is displayed on to a DSO through a $50 \Omega$ terminator as shown in Fig 2. 


\section{Results and discussions}

The variation of laser power as function of input dc current from the ckt of fig 1 is shown in fig 3. The output power from the laser suddenly starts increasing at a particular value of current. Below this, output power is extremely low (this corresponds to the spontaneous emission only). The value of current at which power of the diode laser suddenly increases corresponds to the threshold current for the diode laser. We have observed for the large number of key chain laser the threshold current in the range of 15-25mA.

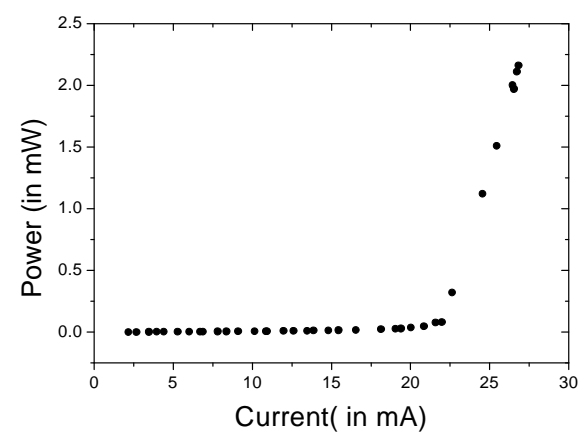

Fig 3. Variation of output power of diode laser with respect to current

The modulated laser out put when a square wave signal is applied from the function generator (Fig 2) is shown in Fig 4a. Trace (i) of fig $4 a$ is from the photodiode signal

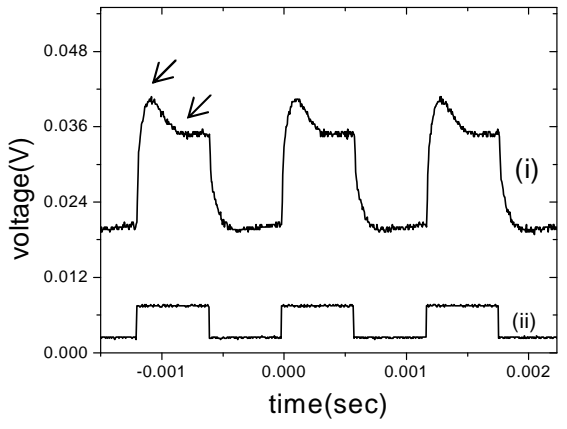

a

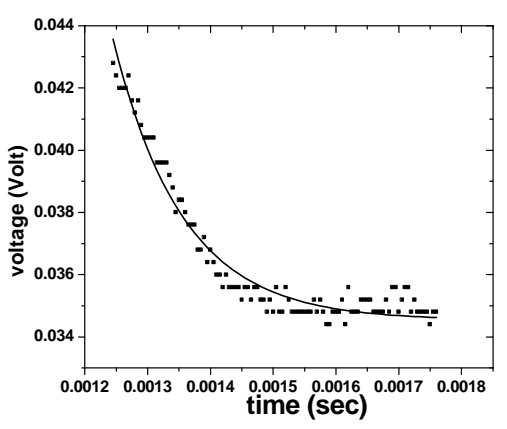

b

Fig 4. a. Laser pulse with respect to the modulating pulse from the function generator, (i) laser pulse, (ii) corresponding pulse from the function generator. b. Exponential fit 
corresponding to laser pulse and trace (ii) is that of from function generator. The exponential fit to the marked portion of trace (i) is shown in Fig $3 \mathrm{~b}$ for the measurement of damping time $t_{0}$. The damping time $t_{o}$ of the relaxation oscillations is given by ${ }^{2}$

$t_{o}=2 \tau / x$

where, $\tau$ is effective life time of upper laser level and $x$ is the amount by which pump rate is above the threshold which was measured by recording the current through the laser diode. The effective lifetime of the upper laser level from the measurement of $t_{o}$ and $x$ were found to be from few microsecond to hundreds of microsecond for various laser diode. The relaxation oscillation frequency $\omega$ is governed by the expression

$\omega=\left[\frac{x-1}{\tau \tau_{c}}-\left(\frac{x}{2 \tau}\right)^{2}\right]^{1 / 2}$

where, $\tau_{c}$ is the cavity photon life time $e^{1,2}$. From eq. 2 it is clear that $x$ has to be set carefully for a given laser system so as to observe the relaxation oscillations. However, damping can always be observed whenever pump is suddenly increased above steady state. The top trace of Fig 5 shows one such relaxation oscillations recorded with the set-up of fig 2. This data was fitted to damped oscillatory function to obtained the relaxation frequency and the cavity life time $\tau_{c}$. We could observe the relaxation oscillations in the range of few $\mathrm{kHz}$ to around $\mathrm{MHz}$ by using large number of locally available low cost diode lasers. The photon life time estimated from the fig 5 was $130 \mu \mathrm{s}$

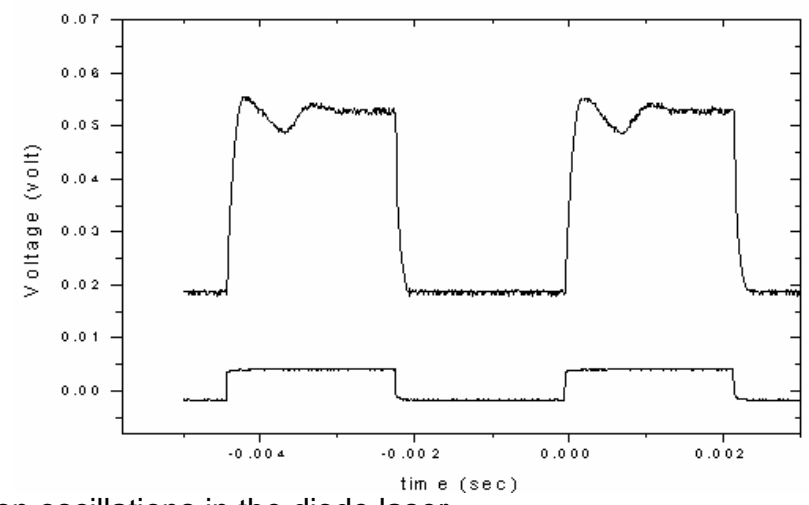

Fig 5. Relaxation oscillations in the diode laser.

Though an analog oscilloscope can also be used for performing the experiment but for the measurement of damping time and oscillations frequency a DSO is recommended.

\section{Conclusion}

We have demonstrated that with the help of simple circuitry and a key chain laser the threshold current, relaxation oscillations damping time and upper laser life time can be easily measured in the teaching laboratory. 


\section{Reference}

1. Shalini Mishra and Alika Khare, 'A simple laboratory Experiment for studying the Relaxation oscillations in a diode laser', Resonance Journal of Science education, 9, 55-66 (2004).

2. O' Svelto, Principle of Lasers, Plenum Press (1998).

3. Alika Khare, 'Pulsed laser operation of a CW laser', Electronics For You, 33, 8889 (2001). 